\title{
microRNA-9 attenuates amyloid $\beta$-induced synaptotoxicity by targeting calcium/calmodulin-dependent protein kinase kinase 2
}

\author{
FEI CHANG, LIN-HONG ZHANG, WU-PING XU, PING JING and PEI-YAN ZHAN \\ Department of Neurology, The Central Hospital of Wuhan, Wuhan, Hubei 430014, P.R. China
}

Received July 3, 2013; Accepted February 18, 2014

DOI: $10.3892 / \mathrm{mmr} .2014 .2013$

\begin{abstract}
The calcium/calmodulin-dependent protein kinase kinase 2, adenosine monophosphate-activated protein kinase (CAMKK2-AMPK) pathway mediated amyloid $\beta 42$ (A $\beta 42$ )-induced synaptotoxicity and blockage of CAMKK2-protected neurons against the effect of $\mathrm{A} \beta 42$. Numerous microRNAs (miRNAs) were downregulated in response to $A \beta 42$, including miR-9, a synapse-enriched miRNA that is decreased in Alzheimer's disease. In the present study the effect of miR-9 on A 342 -triggered CAMKK2-AMPK activation and the synaptotoxic impairment was investigated. A $\beta 42$ oligomers were identified to be capable of inducing CAMKK2-AMPK pathway activation, which was attenuated by miR-9 overexpression. CAMKK2 was predicted to be a target of miR-9 using Pictar and Targetscan 6.2 Bioinformatics' algorithms. A luciferase activity assay and western blot analysis confirmed that miR-9 significantly inhibited CAMKK2 expression. Additionally, overexpression of miR-9 was sufficient to restore $A \beta 42$-induced dendritic spine loss and rescued A $\beta 42$-induced $\tau$ phosphorylation at Ser-262 mediated by the CAMKK2-AMPK pathway. The results of the present study demonstrated that miR-9 attenuated $A \beta$-induced synaptotoxicity by targeting CAMKK2.
\end{abstract}

\section{Introduction}

Alzheimer's disease (AD) is the most prevalent neurodegenerative disease affecting $>25$ million people worldwide. It is characterized by progressive loss of cognitive function resulting in dementia and mortality (1). The pathological hallmarks of AD are extracellular senile plaques (SPs) composed of amyloid $\beta(\mathrm{A} \beta)$ and intracellular neurofibrillary tangles

Correspondence to: Mr. Fei Chang, Department of Neurology, The Central Hospital of Wuhan, 26 Shengli Road, Wuhan, Hubei 430014, P.R. China

E-mail: fei_chang027@163.com

Keywords: amyloid $\beta$, microRNA-9, calcium/calmodulin-dependent protein kinase kinase 2, adenosine monophosphate-activated protein kinase, synapse, Alzheimer's disease consisting of hyperphosphorylated microtubule-associated protein $\tau$ (2). A $\beta$ aggregation and accumulation, derived from sequential cleavage of amyloid precursor protein (APP) mediated by $\beta$ - and $\gamma$-secretases, is the triggering event in the process of AD (2-4). The A $\beta$ oligomer is the main form which mediates the deleterious effect of $A \beta$ as it fibrillates to form SPs. Emerging evidence has indicated the possible interaction between $A \beta$ and $\tau$, and their synergistic effects during AD progression (5). However, the underlying molecular mechanisms linking $A \beta$ and $\tau$ remain poorly understood.

Synaptic dysfunction has also been detected in the brains of patients with AD prior to the appearance of amyloid plaques (6). A $\beta 42$ oligomers have been reported to induce the acute rapid synaptotoxic effect and $\tau$ phosphorylation at Ser-262 by activating the calcium/calmodulin-dependent protein kinase kinase 2, adenosine monophosphate-activated protein kinase (CAMKK2-AMPK) pathway (1). AMPK acts as a metabolic sensor and is an essential regulator of the cellular metabolism. AMPK is activated by an increased intracellular AMP/adenosine triphosphate (ATP) ratio as well as other forms of cellular stress. Once activated, AMPK regulates a variety of biological processes, including cell polarity, apoptosis, cell migration and synaptic plasticity $(7,8)$. Accumulating studies have indicated that AMPK-signaling regulates $\tau$ phosphorylation and amyloidogenesis in the AD pathogenesis (1,9-11). Activated AMPK was observed to markedly enriched in tangle-bearing neurons in patients with AD (12). These observations indicate that AMPK may be involved in the pathogenesis of AD.

microRNAs (miRNAs) are short, non-coding RNAs that inhibit protein expression by binding to specific recognition elements in the 3 ' untranslated region (3'UTR) of target transcripts leading to mRNA translation suppression or mRNA degradation. Currently, >700 miRNAs have been identified, and are essential in a number of cellular processes, including cell polarity, migration, apoptosis and synaptic plasticity (13). miRNA expression profiles in patients with AD and AD animal models have been identified. miRNAs have been reported to regulate $A \beta$ generation, the inflammatory response and neurogenesis in $\mathrm{AD}$ pathogenesis (14).

miR-9 is a synapse-enriched miRNA and was observed to be markedly decreased in patients with AD (15). miR-9 is specifically expressed in the brain and promotes neurogenesis by suppressing the basic helix-loop-helix hairy/enhancer 
of split-1 [E(sp1)] transcription factors Her5 and Her9 expression (16). Schonrock et al reported that miR-9 was downregulated by $A \beta$ treatment and suppressed the expression of a variety of genes $(17,18)$. However, the role of miR-9 during the $A \beta$-induced synaptotoxic effect is poorly understood. In the present study the effect of miR-9 on A 342 -triggered CAMKK2-AMPK activation and the synaptotoxic impairment was investigated.

\section{Materials and methods}

A $\beta 42$ oligomer preparation. A $\beta 42$ oligomer preparation was performed as previously reported (1). Briefly, the $A \beta 42$ peptides (China Peptide, Shanghai, China) were dissolved in hexafluoro-2-propanol (HFIP) for $2 \mathrm{~h}$, and then the HFIP was removed by speed vacuum (Neu-Tec Group Inc., Farmingdale, NY, USA). Dimethylsulfoxide was added to produce a $5 \mathrm{mM}$ solution. This solution was added to cold phenol red-free F12 medium (Invitrogen Life Technologies, New York, NY, USA), incubated at $4^{\circ} \mathrm{C}$ for $24 \mathrm{~h}$ and then centrifuged at $14,000 \mathrm{x}$ g for $10 \mathrm{~min}$ to discard fibrils. The supernatant was kept and used as a source of $\mathrm{A} \beta 42$ oligomers.

RNA extraction and quantitative PCR ( $P P C R)$. The total RNA was collected from cells with TRIzol (Invitrogen Life Technologies), according to the manufacturer's instructions. In total, $1 \mu \mathrm{g}$ RNA was reverse-transcribed into cDNA with a reverse transcription kit (Toyobo, Dalian, China). miRNAs were collected using a microRNA Extraction kit (Tiangen, Beijing, China). Poly(A) was added and $1 \mu \mathrm{g}$ RNA containing miRNAs was reversely transcribed into cDNA. The synthesized cDNAs were amplified using the SYBR qPCR kit (Takara, Dalian, China) on ABI Stepone plus equipment (ABI, Foster City, CA, USA). Expression of CAMKK2 was normalized with GAPDH, and miR-9 and miR-181c levels were normalized with U6 snRNA.

Constructs and luciferase assay. The miR-9 expression construct (catalog no. MmiR-AN0825-AM02) was purchased from GeneCopoeia (Rockville, MD, USA). The possible target positions of the CAMKK2 3'UTR sequences were subcloned into the psiCHECK-2 between XhoI and Not I restriction sites (Promega Corporation, Madison, WI, USA). The position 1898-2358 of CAMKK2 3'UTR was amplified from mouse hippocampus cDNA with the following primers: Sense: 5'-CTCGAGTGCCCGAGTAGGGTAGGCGTG-3' and antisense: 3'-AGCGGCCGCTGAACGAGGCTTGTGCTT-5'. Mutations in the miR-9 binding-sites of CAMKK2 were introduced with a fast whole-plasmid mutation kit (NEB, Ipswich, Canada).

HEK-293 cells were plated onto a 96-well plate. Subsequent to $24 \mathrm{~h}$ incubation, the cells were treated with a cotransfection consisting of $35 \mu \mathrm{l}$ serum-free medium, $0.5 \mu \mathrm{l}$ Lipofectamine 2000, $0.03 \mu \mathrm{g}$ psiCHECK-2-CAMKK2 and $0.1 \mu \mathrm{g}$ miR-9 per well. Renilla luciferase or pEZX-AM02 vector was used as a negative control. After $4 \mathrm{~h}, 100 \mu \mathrm{l}$ serum-containing culture medium was added to the wells. The luciferase activity was examined $48 \mathrm{~h}$ after transfection using the Dual-Luciferase ${ }^{\circledR}$ Reporter 1000 Assay system (Promega Corporation).
Primary neuronal culture and transfection. Primary embryonic E18 hippocampal neurons of the mice were cultured according to a procedure described previously (19). Briefly, the hippocampal neurons were collected and incubated with $5 \mathrm{ml}$ D-Hank's containing $0.25 \%$ trypsin for $15 \mathrm{~min}$ and centrifuged at $1000 \mathrm{x}$ g for $5 \mathrm{~min}$ following addition of $5 \mathrm{ml}$ Dulbecco's modified Eagle's medium with Ham's F12 medium with $10 \%$ fetal bovine serum, the cells were triturated and seeded onto a 60-mm plastic culture dish at a density of $100-200$ neurons $/ \mathrm{mm}^{2}$ and maintained in a humidified incubator at $37^{\circ} \mathrm{C}$ with $5 \% \mathrm{CO}_{2}$ for $4 \mathrm{~h}$. Next, the culture medium was replaced by neurobasal medium with 2\% B27 and the cells were cultured for 18 days. Transfection was performed according to the manufacturer's instructions (Invitrogen Life Technologies), and the ratio of the constructs to Lipofectamine 2000 was 1:2.

AMPK activity assay. Cellular AMPK activity was determined using the SAMS peptide phosphorylation assay kit (Upstate Biotechnology, Lake Placid, NY, USA) according to the manufacturer's instructions. Briefly, the cells were maintained in serum-free medium for $12 \mathrm{~h}$ prior to drug exposure. $\mathrm{A} \beta 42$ oligomers or the scramble control to the final concentration of $1 \mu \mathrm{M}$ were added to the cell culture, and incubated at $37^{\circ} \mathrm{C}$ for $1 \mathrm{~h}$. The culture medium was removed, and the cells were harvested in Tris-HCl supplemented with the protease inhibitors aprotinin, leupeptin, and pepstain A (Roche Diagnostics, Pleasanton CA, USA). The cellular debris were removed following centrifugation at $10,000 \mathrm{x}$ g at $4^{\circ} \mathrm{C}$ for $15 \mathrm{~min}$ and the supernatants were stored at $-70^{\circ} \mathrm{C}$ prior to the AMPK activity assays (20).

Western blot analysis. The cells were harvested and extracted with protein radio-immunoprecipitation assay buffer, supplemented with a cocktail of protease (Roche) and phosphatase (Sigma-Aldrich, SG, Switzerland) inhibitors. Equal quantities of proteins were separated by $10 \%$ sodium dodecyl sulfate-polyacrylamide gel, transferred to polyvinylidene difluoride membranes (Millipore, Billerica, MA, USA) and detected using specific primary antibodies overnight at $4^{\circ} \mathrm{C}$, including pT172 (rabbit monoclonal; Cell Signaling Technology, Inc., San Diego, CA, USA), AMPK (rabbit polyclonal; Cell Signaling Technology, Inc.), CAMKK2 (rabbit polyclonal; Abcam, Cambridge, UK), GAPDH (rabbit monoclonal; Cell Signaling), pS262 (rabbit polyclonal; Abcam) and Tau (mouse monoclonal; Millipore, Hayward, CA, USA). The membranes were incubated with secondary antibodies, conjugated to horseradish peroxidase for $1 \mathrm{~h}$ at $37^{\circ} \mathrm{C}$ and visualized using an enhanced chemiluminescence kit (Pierce, Rockford, IL, USA). The blots were scanned and analyzed by Kodak Digital Science 1D software (Eastman Kodak, Rochester, NY, USA).

Image acquisition and analyses. The images were acquired in 2048x2048 resolution using the A1R laser-scanning confocal microscope (Nikon A1R-si Laser scanning confocal microscope; Nikon, Tokyo, Japan) with the Nikon software NIS-Elements (Nikon, Melville, NY, USA). The dendritic spine density was quantified on branches proximal to the soma.

Statistical analysis. Data were analyzed by using SPSS 16.0 statistical software (SPSS Inc., Chicago, IL, USA), and 
A

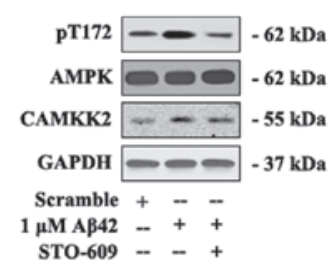

D

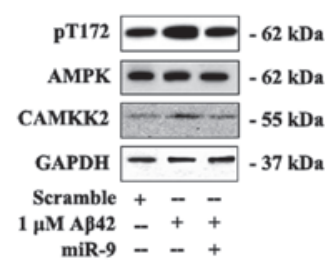

B

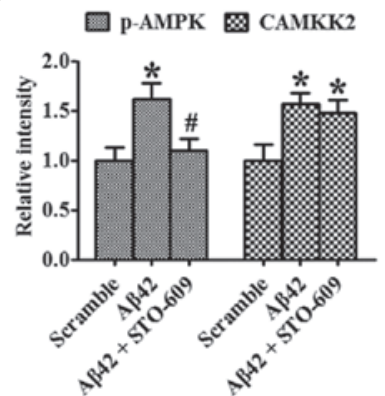

$\mathbf{E}$

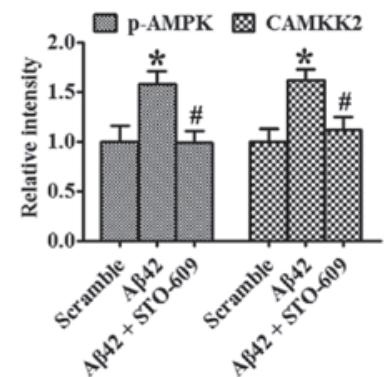

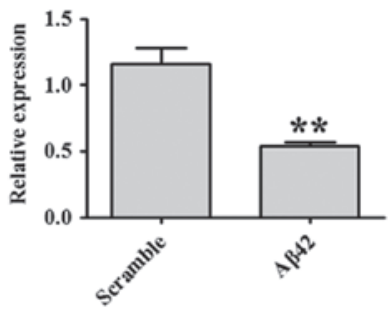

$\mathbf{F}$

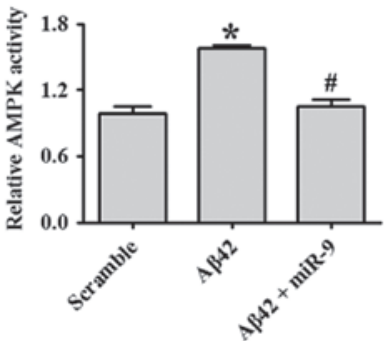

Figure 1. Overexpression of miR-9 inhibited A $\beta 42$ induced AMPK activation. Primary hippocampal neurons isolated from E18 mouse embryos were maintained for $18 \mathrm{DIV}$ and then treated with $1 \mu \mathrm{M} \mathrm{A} \beta 42$ oligomers or the scramble control for $12 \mathrm{~h}$ in the absence or presence of miR-9 overexpression construct or $2.5 \mu \mathrm{M}$ STO-609, an inhibitor of CAMKK2. (A and D) Activation of AMPK was examined by western blot analysis using the phospho-specific T172-AMPK antibody and total AMPK antibody. (B and E) The relative intensity was analyzed. (C) miR-9 expression was determined by quantitative polymerase chain reaction normalized by U6. (F) The AMPK relative activity was assayed by using a commercial kit. Statistical analysis was performed by using one-way analysis of variance followed by Dunnett's post-test. Data were drawn from three independent experiments. " $\mathrm{P}<0.05$ and ${ }^{* *} \mathrm{P}<0.01$, compared with control; ${ }^{\#} \mathrm{P}<0.05$, compared with A 342 group. miR-9, microRNA-9; A $\beta 42$, amyloid $\beta 42$; CAMKK2, calcium/calmodulin-dependent protein kinase kinase 2; AMPK, adenosine monophosphate protein kinase; DIV, days in vitro.

A

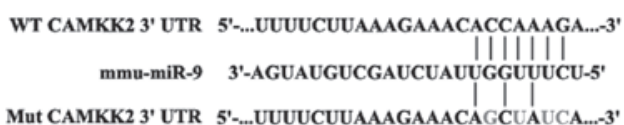

C

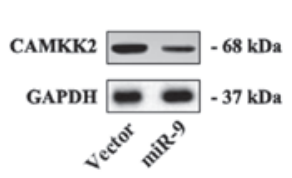

D

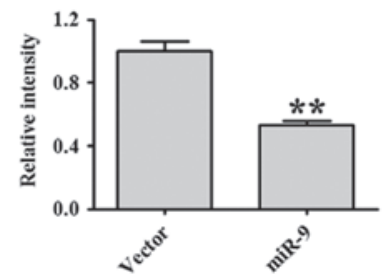

$\mathbf{E}$
B
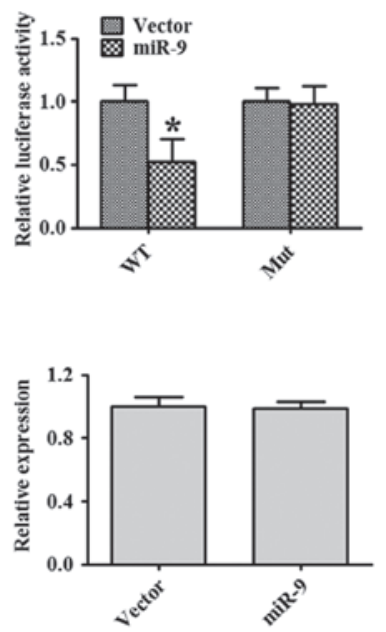

Figure 2. miR-9 suppressed CAMKK2 translation. (A) Schematic diagram of the 3'UTRs of the WT CAMKK2 and mut CAMKK2 luciferase constructs. (B) Dual-luciferase assays were performed in the HEK293 cells. The reporter plasmid containing the WT CAMKK2 3'UTR or the mut CAMKK2 3'UTR was cotransfected with miR-9 overexpression construct or the vector. The Renilla luciferase activity was normalized by the firefly luciferase activity. (C) Primary hippocampal neurons isolated from E18 mouse embryos were maintained for 10 DIV and transfected with miR-9 overexpression construct or the vector CAMKK2 protein level was detected by western blot analysis and (D) the relative intensity was analyzed. (E) CAMKK2 mRNA levels following transfection was measured by quantitative polymerase chain reaction. Statistical analysis was performed by Student's t test. Data were drawn from four separate experiments. " $\mathrm{P}<0.05$ and ${ }^{* * *} \mathrm{P}<0.01$ compared with control. CAMKK2, calcium/calmodulin-dependent protein kinase kinase 2; UTR, untranslated region; WT, wild type; miR-9, microRNA-9; DIV, days in vitro.

one-way analysis of variance with Dunnett's post-test was used for multiple comparisons. Student's t-test was used to determine the differences between the two groups. $\mathrm{P}<0.05$ was considered to indicate a statistically significant difference.

\section{Results}

Overexpression of miR-9 inhibited A 342 -induced $A M P K$ activation. The effect of $\mathrm{A} \beta 42$ oligomers on the activation of 
A

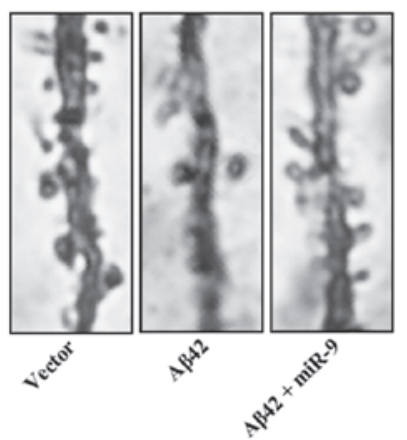

B

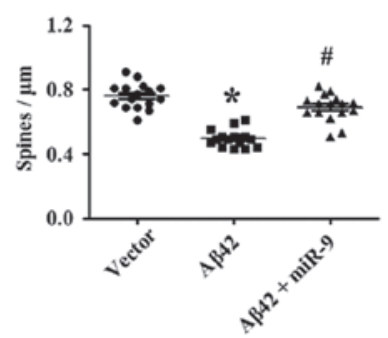

C

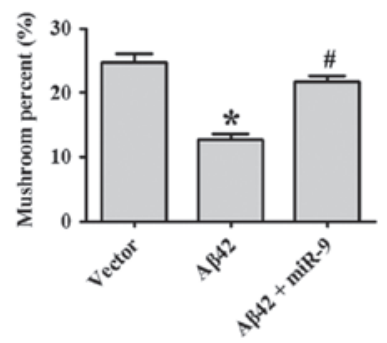

Figure 3. miR-9 rescued A 342 induced synaptotoxicity by targeting CAMKK2. Representative images of secondary dendritic segments of hippocampal neurons obtained from the control, A 342 oligomers with/without miR-9 overexpression construct. (A) Hippocampal neurons were transfected with pEGFP-N1 plasmid at 10 DIV for spine visualization and treated at 18 DIV. (B, C) Spine density and mushroom percentage were measured on 15 dendritic segments per condition. Statistical analysis was performed by using one-way analysis of variance test followed by Dunnett's post-test. Data were drawn from three independent experiments. " $\mathrm{P}<0.05$, compared with control and ${ }^{\#} \mathrm{P}<0.05$, compared with the A $\beta 42$ group. miR-9, microRNA-9; A $\beta 42$, amyloid $\beta 42$; CAMKK2, calcium/calmodulin-dependent protein kinase kinase 2; DIV, days in vitro.

A

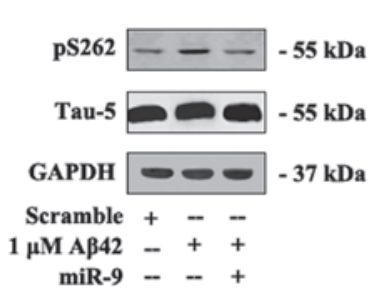

B

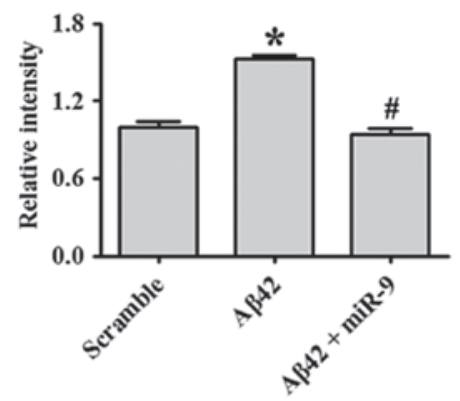

Figure 4. miR-9 attenuated A $\beta 42$ induced $\tau$ phosphorylation by targeting CAMKK2. (A) Hippocampal neurons were transfected at 10 DIV with miR-9 overexpression construct or the vector, treated with $A \beta 42$ oligomers or the scramble control at 20 DIV for $12 \mathrm{~h}$. (B) The relative intensity was analyzed. A statistical analysis was performed by using one-way analysis of variance followed by Dunnett's post-test. Data were drawn from three independent experiments. ${ }^{*} \mathrm{P}<0.05$, compared with control and ${ }^{\#} \mathrm{P}<0.05$, compared with the $\mathrm{A} \beta 42$ group. CAMKK2, calcium/calmodulin-dependent protein kinase kinase 2; miR-9, microRNA-9; DIV, days in vitro.

the CAMKK2-AMPK2 pathway was confirmed in the present study. Days in vitro (DIV)18 primary hippocampal neurons were treated with $1 \mu \mathrm{M} A \beta 42$ oligomers or the scrambled control for $12 \mathrm{~h}$. Activated AMPK, pT172-AMPK and total AMPK were detected by western blot analysis. A $\beta 42$ elevated CAMKK2 expression and A $\beta 42$ treatment also significantly increased the ratio of pT172-AMPK/AMPK. A supplement of STO-609, a specific inhibitor of CAMKK2, attenuated the levels of pT172-AMPK (Fig. 1A and B). Previous studies reported that $A \beta 42$ could decrease the expression of several miRNAs including miR-9. In the present study the expression of miR-9 in primary hippocampal neurons treated with $A \beta 42$ oligomers for $12 \mathrm{~h}$ was measured. A $\beta 42$ markedly inhibited miR-9 expression (Fig. 1C). In order to evaluate the function of miR-9 on the CAMKK2-AMPK pathway activated by $\mathrm{A} \beta 42$ oligomers, miR-9 at DIV 10 was overexpressed and neurons were treated with A $\beta 42$ oligomers at DIV18. miR-9 overexpression was found to substantially eradicate the elevation of CAMKK2 expression and the activation of

AMPK as shown by decreased CAMKK2 and pT172-AMPK levels (Fig. 1D and E). AMPK activity was also examined by the SAMS Peptide Phosphorylation Assay kit (Fig 1D). These data indicated that overexpression of miR-9 inhibited CAMKK2-AMPK activation by A $\beta 42$ oligomers.

miR-9 suppressed CAMKK2 translation. To analyze the potential targets of miR-9, Pictar (http://pictar.mdc-berlin.de/) and Targetscan 6.2 (http://www.targetscan.org/) Bioinformatics' algorithms were used to screen the potential gene. CAMKK2 was identified to be a potential target of miR-9 predicted by the two algorithms. The predicted sequences are shown in Fig. 2A. To test this hypothesis, a wild-type and a mutant of CAMKK2 3'UTR were generated and these sequences were inserted into the luciferase reporter vector. When coexpressed with miR-9, a wild-type reporter revealed significant inhibition (Fig. 2B), while without effect on its mutant (Mut). The mRNA and protein levels of CAMKK2 were also detected. miR-9 significantly decreased the protein level of CAMKK2 (Fig. 2C and D), while it did not affect the mRNA level of CAMKK2 (Fig. 2E). These results demonstrated that miR-9 directly targeted CAMKK2.

miR-9 rescued A $\beta 42$-induced synaptotoxicity by targeting $C A M K K 2$. Previous studies demonstrated that $1 \mu \mathrm{M}$ A $\beta 42$ oligomers induced a significant reduction in dendritic spine density, while it did not affect neuronal viability (1). In addition, the present study tested whether miR-9 overexpression was sufficient to eradicate the synaptotoxic effects of A $\beta 42$ oligomers. As shown in Fig. 3A and quantified in Fig. 3B and C, the results demonstrate that the overexpression of miR-9 restored the reduction in spine density following A $\beta 42$ oligomer application.

miR-9 attenuated A $\beta 42$-induced $\tau$ phosphorylation by targeting $C A M K K 2$. Plaques of $\mathrm{A} \beta$ deposition and tangles formed by hyperphosphorylated microtubule-binding protein $\tau$ were the two major histopathological signatures observed in the brains of AD patients. Although $\mathrm{A} \beta$ and $\tau$ have been extensively investigated independently with regard to their separate 
toxic effects, recent results indicated their potential interactions or synergistic effects during AD progression $(21,22)$. For instance, $\tau$ was observed to mediate $A \beta$ signals to drive ectopic neuronal cell cycle re-entry in AD (23). Recent results had revealed that AMPK is a potent $\tau$ kinase (24). S262 of $\tau$ was a significant target of AMPK, and A $\beta 42$ oligomers increased pS262- $\tau$ phosphorylation by activating AMPK (25). In the present study, overexpression of miR-9 was identified to attenuate $\mathrm{A} \beta 42$-induced $\tau$ phosphorylation by targeting CAMKK2 (Fig. 4A and B).

\section{Discussion}

Loss of synapses begins during the early stages of AD, prior to plaque formation, and progressively affect neuronal activity, leading to cognitive impairment. $A \beta$ oligomers had been hypothesized to contribute to synapse loss, and $\tau$ acted as an essential mediator of $\mathrm{A} \beta$ synaptotoxicity. In vitro and in vivo studies demonstrated that $A \beta 42$ oligomers activated the CAMKK2-AMPK pathway, which phosphorylated $\tau$ on S262 in the microtubule-binding domain inducing dendritic spine loss in hippocampal neurons $(1,24,26)$. Inhibition of CAMKK2, or overexpression of the unphosphorylated mutant of $\tau$ (S262A) eradicated A $\beta 42$ oligomer-induced synaptotoxicity $(1,27)$.

AMPK is a sensor of cellular stress, which maintains energy homeostasis by regulating several metabolic enzyme activities. AMPK is a heterotrimeric Ser/Thr kinase, with a catalytic $\alpha$ subunit and two regulatory subunits, $\beta$ and $\gamma$. Regulation of AMPK activity involved activation by AMP and phosphorylation of the AMPK $\alpha$ subunit at Thr-172 within the activation loop by its upstream kinases. The major upstream kinases included liver kinase B1, in response to increased AMP/ATP ratio, and CAMKK2, in response to elevated intracellular $\mathrm{Ca}^{2+}$ levels (28). By contrast, Thr-172 was dephosphorylated by protein phosphatase-2C to deactivate AMPK. AMPK was widely expressed in mammalian tissues and cell types, including the hippocampus. Epidemiological studies and functional neuroimaging have demonstrated perturbed brain energy metabolism in patients with AD. Perturbation of brain energy metabolism is involved in the neurodegeneration occurring in early stages of $\mathrm{AD}$, and may correlate with early cognitive dysfunction, including increased problems in maintaining $\mathrm{Ca}^{2+}$ homeostasis, decline in glucose uptake, synaptotoxicity and mitochondrial dysfunction (29). AMPK also regulated $\mathrm{A} \beta$ generation through regulating APP processing (30).

miRNAs are significant in a variety of neurological processes, including synaptic plasticity and stress responses. Previous studies of miRNAs in AD demonstrated that numerous miRNAs, including miR-9, miR-124a, miR-125b and miR-132, abundantly expressed in fetal hippocampus were differentially regulated in the aged brain (31). miRNAs were also shown to participate in the pathogenesis of AD. For instance, miR-29a/b-1, miR-195, miR-298 and miR-328 suppressed $A \beta$ generation by inhibiting a $\beta$-amyloid precursor protein-converting enzyme $(13,32,33)$. miR-16, miR-17-5p, miR-20a, miR-106a and miR-106b were reported to regulate APP expression, indicating that variations in miRNA expression may contribute to the alteration in APP expression and A $\beta$ production in the brain during development and disease $(34,35)$.
Conversely, $A \beta$ also induced abnormal expression of miRNAs, including miR-9, miR-21 and miR-181c (18). However, the role of miRNAs in $A \beta$ induced synaptotoxic effect remains poorly understood.

miR-9, a brain-specific and synapse enriched miRNA, was significantly decreased in patients with AD (36). miR-9 was initially identified as a crucial regulator of the development and physiology of the nervous system in numerous organisms, including Drosophila and mammals (16). miR-9 regulated the proliferation, differentiation and migration of neural stem cells by controlling hairy/E (spl1) (37). miR-9 was able to improve neurite outgrowth by targeting Forkhead box protein P2 (37). Although miR-9 was decreased in response to A $\beta 42$, the potential role of miR-9 in A $\beta 42$ induced synaptotoxicity remains unknown. In the present study overexpression of miR-9 was found to be capable of attenuating $A \beta 42$-induced CAMKK2-AMPK pathway activation, rescuing A $\beta 42$-induced dendritic spine density loss and eradicating A $\beta 42$-induced $\tau$ phosphorylation on S262 partially by targeting CAMKK2 .

In conclusion, miR-9 was shown to antagonize the A $\beta 42$-induced synaptotoxic effect by targeting CAMKK2, which may provide a novel strategy for AD therapy.

\section{References}

1. Mairet-Coello G, Courchet J, Pieraut S, Courchet V, Maximov A and Polleux F: The CAMKK2-AMPK kinase pathway mediates the synaptotoxic effects of $\mathrm{A} \beta$ oligomers through tau phosphorylation. Neuron 78: 94-108, 2013.

2. Wang JZ and Liu F: Microtubule-associated protein tau in development, degeneration and protection of neurons. Prog Neurobiol 85: 148-175, 2008.

3. Vanitallie TB: Preclinical sporadic Alzheimer's disease: target for personalized diagnosis and preventive intervention. Metabolism 62 (Suppl 1): S30-S33, 2013.

4. Wang JF, Lu R and Wang YZ: Regulation of $\beta$ cleavage of amyloid precursor protein. Neurosci Bull 26: 417-427, 2010.

5. Jin M, Shepardson N, Yang T, Chen G, Walsh D and Selkoe DJ: Soluble amyloid beta-protein dimers isolated from Alzheimer cortex directly induce Tau hyperphosphorylation and neuritic degeneration. Proc Natl Acad Sci USA 108: 5819-5824, 2011.

6. Palop JJ, Chin J, Roberson ED, et al: Aberrant excitatory neuronal activity and compensatory remodeling of inhibitory hippocampal circuits in mouse models of Alzheimer's disease. Neuron 55: 697-711, 2007.

7. Yuan HX, Xiong Y and Guan KL: Nutrient sensing, metabolism, and cell growth control. Mol Cell 49: 379-387, 2013.

8. Nakano A and Takashima S: LKB1 and AMP-activated protein kinase: regulators of cell polarity. Genes Cells 17: 737-747, 2012.

9. Salminen A, Kaarniranta K, Haapasalo A, Soininen H and Hiltunen M: AMP-activated protein kinase: a potential player in Alzheimer's disease. J Neurochem 118: 460-474, 2011.

10. Park H, Kam TI, Kim Y, et al: Neuropathogenic role of adenylate kinase- 1 in $A \beta$-mediated tau phosphorylation via AMPK and GSK3 $\beta$. Hum Mol Genet 21: 2725-2737, 2012.

11. Kim J, Park YJ, Jang Y and Kwon YH: AMPK activation inhibits apoptosis and tau hyperphosphorylation mediated by palmitate in SH-SY5Y cells. Brain Res 1418: 42-51, 2011.

12. Vingtdeux V, Davies P, Dickson DW and Marambaud P: AMPK is abnormally activated in tangle- and pre-tangle-bearing neurons in Alzheimer's disease and other tauopathies. Acta Neuropathol 121: 337-349, 2011.

13. Fernandez-Hernando C, Ramírez CM, Goedeke L and Suárez Y: MicroRNAs in metabolic disease. Arterioscler Thromb Vasc Biol 33: 178-185, 2013.

14. Delay C, Mandemakers W and Hebert SS: MicroRNAs in Alzheimer's disease. Neurobiol Dis 46: 285-290, 2012.

15. Hébert SS, Horré K, Nicolaï L, et al: Loss of microRNA cluster miR-29a/b-1 in sporadic Alzheimer's disease correlates with increased BACE1/beta-secretase expression. Proc Natl Acad Sci USA 105: 6415-6420, 2008. 
16. Leucht C, Stigloher C, Wizenmann A, Klafke R, Folchert A and Bally-Cuif L: MicroRNA-9 directs late organizer activity of the midbrain-hindbrain boundary. Nat Neurosci 11: 641-648, 2008.

17. Schonrock N, Humphreys DT, Preiss T and Götz J: Target gene repression mediated by miRNAs miR-181c and miR-9 both of which are down-regulated by amyloid- $\beta$. J Mol Neurosci 46: 324-335, 2012

18. Schonrock N, Ke YD, Humphreys D, et al: Neuronal microRNA deregulation in response to Alzheimer's disease amyloid-beta. PLoS One 5: e11070, 2010.

19. Zhu LQ, Zheng HY, Peng CX, et al: Protein phosphatase A facilitates axonogenesis by dephosphorylating CRMP2. J Neurosci 30: 3839-3848, 2010.

20. Rencurel F, Foretz M, Kaufmann MR, et al: Stimulation of AMP-activated protein kinase is essential for the induction of drug metabolizing enzymes by phenobarbital in human and mouse liver. Mol Pharmacol 70: 1925-1934, 2006.

21. Handoko M, Grant M, Kuskowski M, et al: Correlation of specific amyloid- $\beta$ oligomers with tau in cerebrospinal fluid from cognitively normal older adults. JAMA Neurol 5: 594-599, 2013.

22. Gao L, Tian S, Gao H and Xu Y: Hypoxia increases $A \beta$-induced tau phosphorylation by calpain and promotes behavioral consequences in AD transgenic mice. J Mol Neurosci 51: 128-147, 2013.

23. Seward ME, Swanson E, Norambuena A, et al: Amyloid- $\beta$ signals through tau to drive ectopic neuronal cell cycle re-entry in Alzheimer's disease. J Cell Sci 126: 1278-1286, 2013.

24. Thornton C, Bright NJ, Sastre M, Muckett PJ and Carling D: AMP-activated protein kinase (AMPK) is a tau kinase, activated in response to amyloid $\beta$-peptide exposure. Biochem $\mathrm{J} 434$ 503-512, 2011.

25. Yoshida H and Goedert M: Phosphorylation of microtubule-associated protein tau by AMPK-related kinases. J Neurochem 120 165-176, 2012.

26. Moolman DL, Vitolo OV, Vonsattel JP and Shelanski ML: Dendrite and dendritic spine alterations in Alzheimer models J Neurocytol 33: 377-387, 2004.
27. Manczak M and Reddy PH: Abnormal interaction of oligomeric amyloid- $\beta$ with phosphorylated tau: implications to synaptic dysfunction and neuronal damage. J Alzheimers Dis 36: 285-295, 2013.

28. Viollet B, Lantier L, Devin-Leclerc J, et al: Targeting the AMPK pathway for the treatment of Type 2 diabetes. Front Biosci (Landmark Ed) 14: 3380-3400, 2009.

29. Cai Z, Yan LJ,Li K, Quazi SH and Zhao B: Roles of AMP-activated protein kinase in Alzheimer's disease. Neuromolecular Med 14: $1-14,2012$.

30. Lu J, Wu DM,Zheng YL, et al: Quercetin activates AMP-activated protein kinase by reducing $\mathrm{PP} 2 \mathrm{C}$ expression protecting old mouse brain against high cholesterol-induced neurotoxicity. J Pathol 222: 199-212, 2010

31. Lukiw WJ: Micro-RNA speciation in fetal, adult and Alzheimer's disease hippocampus. Neuroreport 18: 297-300, 2007.

32. Ai J, Sun LH, Che H, et al: MicroRNA-195 protects against dementia induced by chronic brain hypoperfusion via its anti-amyloidogenic effect in rats. J Neurosci 33: 3989-4001, 2013

33. Boissonneault V, Plante I, Rivest S and Provost P: MicroRNA-298 and microRNA-328 regulate expression of mouse beta-amyloid precursor protein-converting enzyme 1. J Biol Chem 284: 1971-1981, 2009.

34. Liu W, Liu C, Zhu J, et al: MicroRNA-16 targets amyloid precursor protein to potentially modulate Alzheimer's-associated pathogenesis in SAMP8 mice. Neurobiol Aging 33: 522-534, 2012.

35. Hébert SS, Horré K, Nicolaï L, et al: MicroRNA regulation of Alzheimer's Amyloid precursor protein expression. Neurobiol Dis 33: 422-428, 2009.

36. Cogswell JP, Ward J, Taylor IA, et al: Identification of miRNA changes in Alzheimer's disease brain and CSF yields putative biomarkers and insights into disease pathways. J Alzheimers Dis 14: 27-41, 2008

37. Tan SL, Ohtsuka T, González A and Kageyama R: MicroRNA9 regulates neural stem cell differentiation by controlling Hes1 expression dynamics in the developing brain. Genes Cells 17: 952-961, 2012. 